\title{
Simulation and Experimental Studies on Perfect Tracking Optimal Control of an Electrohydraulic Actuator System
}

\author{
R. Ghazali, ${ }^{1,2}$ Y. M. Sam, ${ }^{2}$ M. F. Rahmat, ${ }^{2}$ Zulfatman, ${ }^{2}$ and A. W. I. M. Hashim² \\ ${ }^{1}$ Department of Mechatronic and Robotic Engineering, Faculty of Electrical and Electronic Engineering, \\ Universiti Tun Hussein Onn Malaysia, Batu Pahat, 86400 Johor, Malaysia \\ ${ }^{2}$ Department of Control and Instrumentation Engineering, Faculty of Electrical Engineering, Universiti Teknologi Malaysia, \\ Skudai, 81310 Johor, Malaysia \\ Correspondence should be addressed to R. Ghazali,rozaimi_85@yahoo.com
}

Received 30 April 2011; Revised 12 October 2011; Accepted 29 October 2011

Academic Editor: Mohamed Zribi

Copyright ( $) 2012$ R. Ghazali et al. This is an open access article distributed under the Creative Commons Attribution License, which permits unrestricted use, distribution, and reproduction in any medium, provided the original work is properly cited.

This paper presents a perfect tracking optimal control for discrete-time nonminimum phase of electrohydraulic actuator (EHA) system by adopting a combination of feedback and feedforward controller. A linear-quadratic regulator (LQR) is firstly designed as a feedback controller, and a feedforward controller is then proposed to eliminate the phase error emerged by the LQR controller during the tracking control. The feedforward controller is developed by implementing the zero phase error tracking control (ZPETC) technique in which the main difficulty arises from the nonminimum phase system with no stable inverse. Subsequently, the proposed controller is performed in simulation and experimental studies where the EHA system is represented in discrete-time model that has been obtained using system identification technique. It also shows that the controller offers better performance as compared to conventional PID controller in reducing phase and gain error that typically occurred in positioning or tracking systems.

\section{Introduction}

In recent years, electrohydraulic actuator (EHA) system has attracted a great interest in industrial engineering as an actuator for high performance and precision positioning applications. With a compact size and design, EHA system is capable of generating high forces in fast response time and produced great durability in particular for heavy engineering system [1]. By utilizing the advantages of EHA system, different applications such as aircrafts [2], manufacturing machines [3-5], fatigue testing [6], hydraulic excavator [7], sheet metal forming process [8], and automotive applications [9-11] established that the actuator system can be more well known and crucial nowadays. Therefore, a feedback tracking control is always required in designing the high-performance for tracking and positioning EHA system.

Various types of feedback controller ranging from linear to nonlinear types are widely implemented and published among academia and researchers for position tracking control of EHA system. The increasing numbers of works dealing with EHA system over the past decades involved a linear control, intelligent control, and nonlinear control approaches such as neural network (NN) [12], self-tuning fuzzy PID $[13,14]$, model reference adaptive control (MRAC) $[15,16]$, generalized predictive control (GPC) [17], and sliding mode control [18]. Most of tracking control system is typically necessary in minimizing gain and phase error from the output to the desired trajectory. This requirement can be achieved by introducing a feedforward controller to eliminate the zeros and poles in the closed loop system. The main objective in that control scheme is to drive the actuator to follow perfectly the desired trajectory and minimize the phase lag that is regularly caused by feedback loop in the closed-loop system.

Generally, a zero phase tracking error can be achieved mathematically by the synthesized inverse model of the closed-loop feedback system in the feedforward design. Thus, a perfect tracking control strategy can be introduced to eliminate phase error, and high-performance tracking can be developed. An optimal feedback controller basically is needed to reduce the disturbance effect from the varying in 


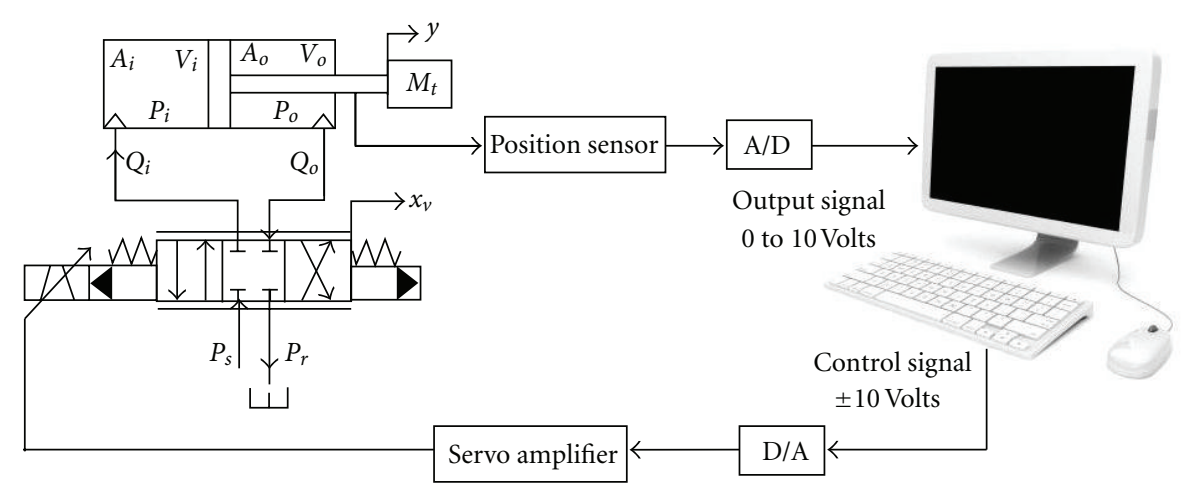

FIGURE 1: Electrohydraulic actuator system.

plant parameters. Several works have been done over the past decades using the direct inverse of the plant model. As an example, sliding mode control is proposed in the feedback design, and the direct inverse of the closed-loop system model is taken in the feedforward design. The robust perfect tracking control is implemented in [19] particularly for minimum phase systems and has been applied experimentally to the drive motor. Direct inverse of the plant can be performed since the zeros of polynomial are stable. However, the feedforward controller is unable to design if the class of systems with disturbance considered is a nonminimum phase which presents zeroes outside the unit circle. For nonminimum phase system, the main difficulty arises from these systems which have no stable inverse. The zeros presented outside the unit circle will be unstable poles during the inversion process.

Direct inverse of the closed-loop systems will cause unstable phenomenon in the output of the feedforward controller, and this is adverse in designing the feedforward control which caused an uncontrollable system. It is established that in [20] the minimum phase system may become nonminimum phase system for sufficiently fast sampling time. One of the most successful feedforward controller designs is the zero phase error tracking controller (ZPETC), which has wide application in advanced manufacturing equipment particularly for nonminimum phase discretetime system [21]. By implementing the ZPETC method, the unstable controller can be designed appropriately by an approximation of the inverse plant, and the transfer function from the reference input to the output is approximately unity. The ZPETC method that was first proposed in [21] had attracted many researchers as one of the solutions to nonminimum phase system in feedforward control strategy. However, the gain error is unable to be eliminated by this method, and also good tracking performance cannot be achieved if the feedback controller is not robust due to varying in parameters, uncertainties, and disturbances. Another method is also proposed in [22] without a factorization of the nonminimum phase systems.

In this study, a perfect tracking control strategy is implemented for a discrete-time nonminimum phase systems by adopting optimal control which is linear-quadratic regulator
(LQR) as a feedback controller. With this control strategy, a state feedback controller such as LQR can be realized for a single-output feedback problem. Therefore, a comparison with the PID controller as an output feedback controller can be made with the proposed controller. An extensive simulation study is conducted first before the implementation of the perfect tracking control strategy. The perfect tracking control strategy is then applied experimentally to the EHA system that is represented in discrete-time model where the discrete model is obtained experimentally using system identification method.

\section{Electrohydraulic Actuator System}

Basic idea of perfect tracking control strategy is to achieve a unity transfer function from the desired trajectory to the output of the systems. Thus, the feedforward controller can be designed by making the closed-loop system transfer function in an inverse arrangement. Furthermore, the phase error can be eliminated in the tracking control system. Generally, modeling of EHA system is required in designing the proposed control strategy. A complete mathematical model with internal leakage and actuator leakage and friction model have been discussed lately in [23] with intelligent control strategy. However, the physical models derived in that simulation study are highly complex and difficult to utilize in some of the control designs especially in the industrial field. Most of the parameters involved in that mathematical model are usually not available in the manufacturer's datasheet and vastly vary with time due to its nonlinearity. Besides, the parameters are affected by the hydraulic oil temperature, supply pressure changes, and aging. A mathematical model of EHA system in Figure 1 can be developed by neglecting these nonlinearities such as internal or external leakage and dynamics of the valve as explained in [12]. In servo valve design, the dynamics of the valve can be approximated as a single gain where the spool valve structure is assumed as critical center and symmetrical. The equation relates the input signal $u$ (either voltage or current) and servo valve gain $K_{v}$, and the spool valve position $x_{v}$ is given by

$$
x_{v}=K_{v} u \text {. }
$$


Thus, the dynamics of the EHA system for the total oil flow $Q_{L}$ are derived from a Taylor series linearization by the following equation

$$
Q_{L}=K_{q} u-K_{c} P_{L}
$$

where $K_{q}=$ flow-gain coefficient and $K_{c}=$ flow-pressure coefficient.

Defining the load pressure, $P_{L}$, as the pressure across the actuator piston, its derivative is given by the total oil load flow $Q_{L}$ through the actuator divided by the fluid capacitance as given by

$$
\dot{P}_{L}=\frac{4 \beta_{e}}{V_{t}}\left(Q_{L}-C_{\mathrm{tp}} P_{L}-A_{p} \dot{y}\right),
$$

where $\beta_{e}$ is the bulk modulus, $V_{t}$ is the total compressed oil volume, $C_{\mathrm{tp}}$ is the total leakage coefficient, $A_{p}$ is the surface area of the piston, and $y$ is the position of the piston. The force of the actuator $F_{a}$ that generates from a total mass $M_{t}$ attached to the end of the piston can be determined as

$$
F_{a}=A_{p} P_{L}=M_{t} \ddot{y} .
$$

Substituting (2) and (3) into the derivative of (4) and taking a Laplace transform yield

$$
\frac{Y(s)}{U(s)}=\frac{K \omega_{n}^{2}}{s\left(s^{2}+2 \xi \omega_{n} s+\omega_{n}^{2}\right)},
$$

where

$$
\begin{gathered}
K=\frac{K_{q} K_{v}}{A_{p}}, \quad \omega_{n}=A_{p} \sqrt{\frac{4 \beta_{e}}{V_{t} M_{t}}}, \\
\xi=\frac{\sqrt{4 \beta_{e} M_{t} / V_{t}}\left(K_{c}+C_{\mathrm{tp}}\right)}{2 A_{p}} .
\end{gathered}
$$

Open-loop transfer function of the EHA system relates between the control signal from the computer as a controller and position of the hydraulic actuator. The corresponding discrete-time model is followed by transforming the continuous-time model in (5) with zero-order hold is expressed as

$$
G(z)=\frac{y(k)}{u(k)}=\frac{b_{1} z^{2}+b_{2} z+b_{3}}{z^{3}+a_{1} z^{2}+a_{2} z+a_{3}} .
$$

Linearization of EHA system has been studied and employed over the past decades to encounter the nonlinearities subsists in the modeling process [12]. There are numerous researchers who used that linear model in either continuous time or discrete time in their proposed control strategy [12, 16-18]. Most of the modeling approaches for discrete-time model that have been implemented in previous researches are developed from first principle or physical laws. Although the importance of physical modeling is always considered in the controller design, but in real implementation, validation of the physical plant model is necessary in optimizing the use of controller that is commonly designed via computer simulation. Furthermore,

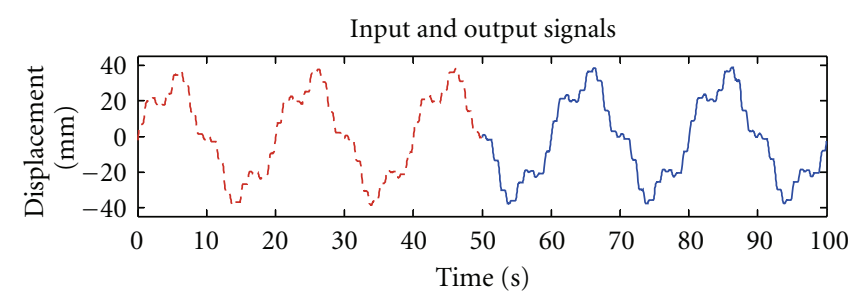

(a)

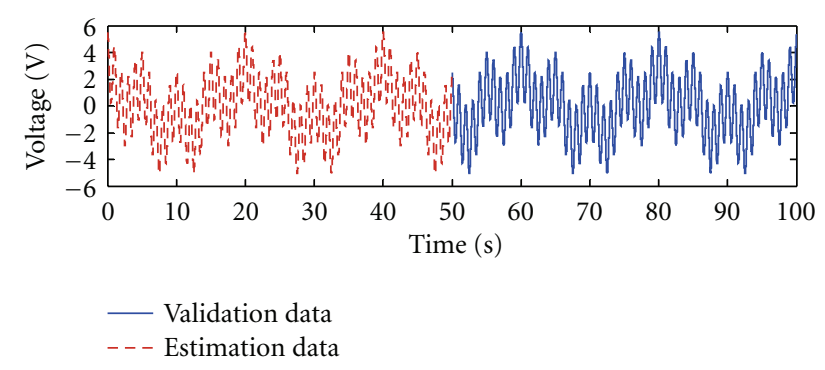

(b)

FIGURE 2: Input-output signals for system identification.

any changes in the EHA system's parameter may reduce the controller performance and the desired specification possibly not achieved. Therefore, system identification technique is needed to determine the nominal model of the EHA system.

System identification was performed first in order to determine the sufficient model order and validity of the linear discrete-time model that has been derived after linearization procedure. The measured signals must be analyzed and preprocessed before the estimation and validation process. The data is divided into two sets where one set is used for estimation process and the other is used to validate the obtained model. A model validation process using an independent data set also is called the cross-validation. A set of data that consists of the input voltage and actuator displacement as shown in Figure 2 was observed for a 100second experiment with 5 milliseconds of sampling time under the off-line model identification.

Various authors have used mean-square error (MSE) [24], root-mean-square error (RMSE) [25, 26], and prediction error [27] for model validation. The performance indices in the validation results can be more conclusive and comparable by using different approaches. Several model orders also have been tested in the experiment study. The main purpose in modelling using system identification approach is to obtain a parsimonious model where the smallest number of parameters or model orders should be preferred in representing a dynamic system [20]. Figure 3 shows the estimated output for the EHA system, while the analysis of the validation process is tabulated in Table 1.

From Table 1, it can be observed that the higher order gives better performance in the observations of simulated outputs. Among the analysis of the different model order, a third-order model appears to be suitable for the system since there is no significant improvement in the model order increment in terms of percentage of fit, final prediction error, and loss of function criterions. Then, correlation analysis 


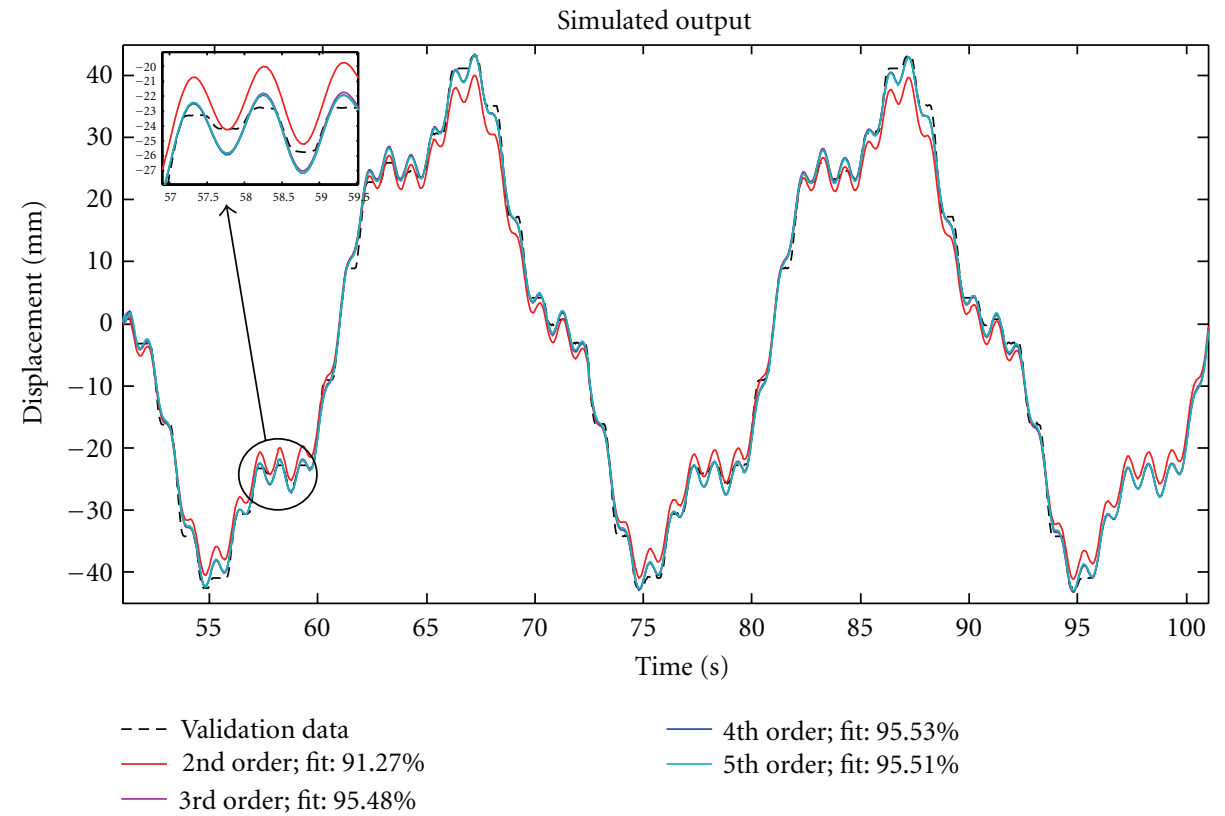

Figure 3: Model validation using best fitting criterion.

TABLe 1: System identification analysis.

\begin{tabular}{lccc}
\hline Model order & $\begin{array}{c}\text { Percentage of fit } \\
(\%)\end{array}$ & $\begin{array}{c}\text { Final prediction } \\
\text { error (FPE) }\end{array}$ & Loss function \\
\hline 2nd order & $91.27 \%$ & 0.0325882 & 0.0323295 \\
3rd order & $95.48 \%$ & 0.0230747 & 0.0228011 \\
4th order & $95.53 \%$ & 0.0217047 & 0.0213629 \\
5th order & $95.51 \%$ & 0.0215606 & 0.0211378 \\
\hline
\end{tabular}

of the residuals is performed as a final procedure in validation process. Residuals are differences between the onestep-predicted output from the estimated model and the measured output from the validation data set. Therefore, residuals can be considered as the portion of the validation data not presented by the model.

Residual analysis in the validation process consists of the whiteness test and the independence test. These tests will indicate the validity of the estimated model. In whiteness test analysis, a good model has the residual autocorrelation function inside the confidence interval and indicating that the residuals are uncorrelated. For independence test criteria, a good model has residuals uncorrelated with past inputs. The residual analysis of third-order model is shown in Figure 4. Thus, the following discrete-time model was identified for the third-order system as shown in (8). The model is found to be as a nonminimum phase model by the zero-pole plot as depicted in Figure 5,

$$
G(z)=\frac{y(k)}{u(k)}=\frac{-0.03093 z^{2}+0.3836 z-0.2738}{z^{3}-1.887 z^{2}+1.056 z-0.1695} .
$$

\section{Optimal Control}

A state space representation is needed in designing the optimal control. The transfer function derived from the EHA system can be transformed in control canonical form as in (9) and (10),

$$
x(k+1)=A x(k)+B u(k)
$$

$$
y(k)=C x(k)
$$

where

$$
\begin{gathered}
A=\left[\begin{array}{ccc}
-a_{1} & -a_{2} & -a_{3} \\
1 & 0 & 0 \\
0 & 1 & 0
\end{array}\right], \quad B=\left[\begin{array}{lll}
1 & 0 & 0
\end{array}\right]^{T}, \\
C=\left[\begin{array}{lll}
b_{1} & b_{2} & b_{3}
\end{array}\right] .
\end{gathered}
$$

Linear-quadratic regulator (LQR) is a part of optimal control strategy which has been widely developed and utilized in various applications. Optimal control is the standard method for solving dynamic optimization problems, when those problems are expressed in continuous time. In this approach, it is advantageous using an optimal control which is LQR to design the feedback control where the method is well known of its robustness towards disturbances and uncertainties. For a discrete-time state-space model in (9), by minimizing the cost function in (12), the LQR gain can be determined as follows:

$$
J=\sum x^{\prime} Q x+u^{\prime} R u+2 x^{\prime} N u
$$


The matrix $N$ is set to zero when omitted. Also returned are the solutions that associated algebraic Riccati equation and the closed-loop eigenvalues. The closed-loop transfer function incorporating the LQR controller can be derived as follows:

$$
\begin{gathered}
u(k)=-K x(k), \\
x(k+1)=(A-B K) x(k), \\
A_{\mathrm{cl}}=A-B K, \\
x(k+1)=A_{\mathrm{cl}} x(k)+B r(k), \\
\frac{y(k)}{r(k)}=C\left(z I-A_{\mathrm{cl}}\right)^{-1} B=G_{\mathrm{cl}}(z) .
\end{gathered}
$$

Based on identified discrete-time model of EHA system in (9), the system matrix $A$ and input matrix $B$ are used to design the LQR. By letting

$$
\mathrm{Q}=\left[\begin{array}{ccc}
1 \times 10^{-4} & 0 & 0 \\
0 & 1 \times 10^{-3} & 0 \\
0 & 0 & 3 \times 10^{-3}
\end{array}\right], \quad R=1,
$$

and minimizing the cost function in (12), the LQR gain is

$$
K=\left[\begin{array}{lll}
0.1849 & -0.1556 & 0.0291
\end{array}\right] .
$$

Then, the closed-loop system matrix can be determined as

$$
A_{\mathrm{cl}}=\left[\begin{array}{ccc}
1.7022 & -0.9009 & 0.1404 \\
1 & 0 & 0 \\
0 & 1 & 0
\end{array}\right]
$$

Therefore, the closed-loop transfer function can be represented as

$$
\begin{aligned}
G_{\mathrm{cl}}(z) & =\frac{y(k)}{r(k)}=C\left(z I-A_{\mathrm{cl}}\right)^{-1} B \\
& =\frac{-0.03093 z^{2}+0.3836 z-0.2738}{z^{3}-1.7022 z^{2}+0.9009 z-0.1404} .
\end{aligned}
$$

The feedforward control can be designed by letting the inverse of closed-loop transfer function with LQR controller as

$$
G_{\mathrm{ff}}(z)=\left[G_{\mathrm{cl}}(z)\right]^{-1} .
$$

It can be observed from the closed-loop transfer function that the numerator which represents the zeroes in the system is dominated by the open-loop system that caused a nonminimum phase problem. The problem of inversion occurred when the $G_{\mathrm{cl}}(z)$ have zeros outside the unit circle where the nonminimum phase of the open-loop EHA system has influenced the closed-loop system dynamics. Direct inverse of the $G_{\mathrm{cl}}(z)$ will cause unstable condition in $G_{\mathrm{fff}}(z)$. Therefore, the method in [21] will be used in developing the feedforward controller to avoid the instability problem.

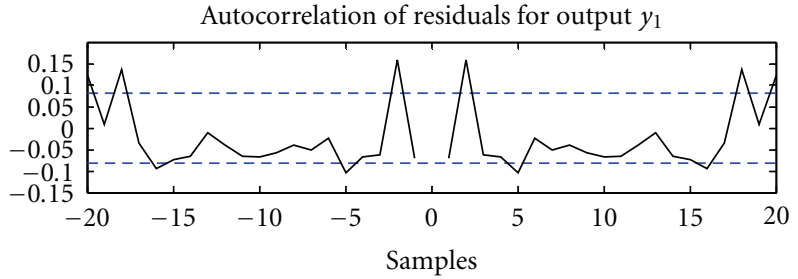

(a)

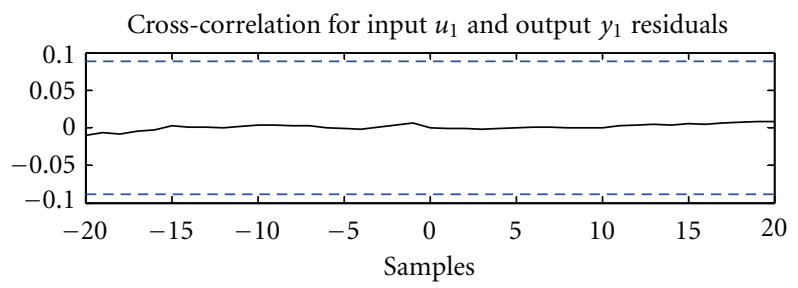

(b)

FIgURE 4: Residual analysis.

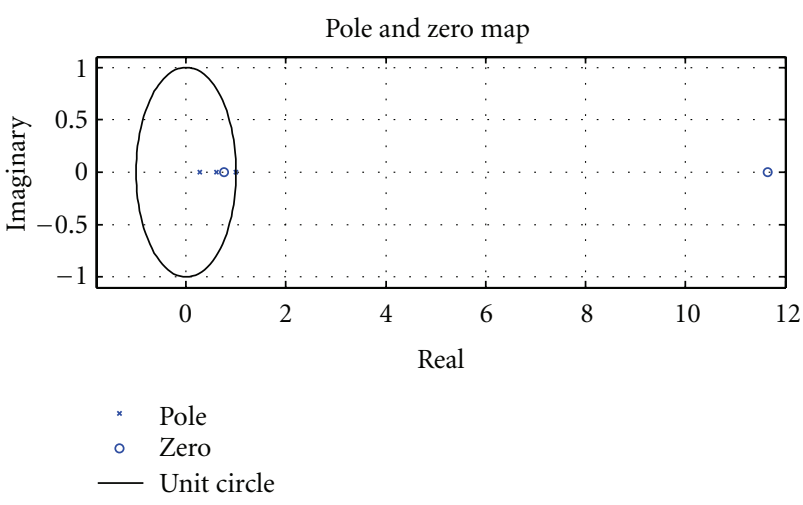

Figure 5: Pole-zero plot for nonminimum EHA system.

\section{Feedforward Controller Design for Nonminimum Phase EHA System}

Since the direct inverse of the closed-loop transfer function is unfeasible, the approximation of the feedforward controller can be implemented to represent the inverse of closed-loop system and cancel the poles and zeros. The direct inversion of closed-loop system can be represented as

$$
\begin{aligned}
G_{\mathrm{ff}}(z) & =\left[G_{\mathrm{cl}}(z)\right]^{-1}=\frac{D_{\mathrm{cl}}(z)}{N_{\mathrm{cl}}(z)} \\
& =\frac{z^{3}-1.7022 z^{2}+0.9009 z-0.1404}{-0.03093 z^{2}+0.3836 z-0.2738} .
\end{aligned}
$$

The block diagram as shown in Figure 6 described the approximation of feedforward control design for nonminimum phase systems using ZPETC strategy. The proposed method can be divided into three blocks which gain compensation filter, phase compensation filter, and its stable inverse. From (19), one of the poles in the feedforward controller is 
outside in the unit circle. In the ZPETC design, (19) can be represented in delay form

$$
\begin{aligned}
G_{\mathrm{ff}}\left(z^{-1}\right) & =\frac{D_{\mathrm{cl}}\left(z^{-1}\right)}{N_{\mathrm{cl}}\left(z^{-1}\right)} \\
& =\frac{1-1.7022 z^{-1}+0.9009 z^{-2}-0.1404 z^{-3}}{-0.03093 z^{-1}+0.3836 z^{-2}-0.2738 z^{-3}} .
\end{aligned}
$$

The numerator of the closed-loop systems can be factorized in the feedforward controller,

$$
\begin{aligned}
G_{\mathrm{ff}}\left(z^{-1}\right) & =\frac{D_{\mathrm{cl}}\left(z^{-1}\right)}{N_{\mathrm{cl}}^{+}\left(z^{-1}\right) N_{\mathrm{cl}}^{-}\left(z^{-1}\right)} \\
& =\frac{1-1.7022 z^{-1}+0.9009 z^{-2}-0.1404 z^{-3}}{-0.0309 z^{-1}\left(1-0.76 z^{-1}\right)\left(1-11.654 z^{-1}\right)},
\end{aligned}
$$

where

$$
\begin{gathered}
N_{\mathrm{cl}}^{+}\left(z^{-1}\right)=-0.0309 z^{-1}\left(1-0.76 z^{-1}\right), \\
N_{\mathrm{cl}}^{-}\left(z^{-1}\right)=1-11.654 z^{-1} .
\end{gathered}
$$

Based on the proposed method, stable inverse can be stated as the following equation:

$$
\begin{aligned}
G_{\mathrm{ffl}}\left(z^{-1}\right) & =\frac{D_{\mathrm{cl}}\left(z^{-1}\right)}{N_{\mathrm{cl}}^{+}\left(z^{-1}\right)} \\
& =\frac{1-0.6798 z^{-1}-0.2405 z^{-2}+0.1350 z^{-3}}{-0.0309 z^{-1}\left(1-0.76 z^{-1}\right)} .
\end{aligned}
$$

In advanced form, the phase compensation filter can be described as

$$
\mathrm{G}_{\mathrm{ff} 2}(z)=z^{d} N_{\mathrm{cl}}^{-}(z)=z(1-11.654 z),
$$

where $d=1$.

And the gain compensation filter is

$$
G_{\mathrm{ff3}}=\frac{1}{\left[N_{\mathrm{cl}}^{-}(1)\right]^{2}}=\frac{1}{[1-11.654(1)]^{2}}=0.00881 .
$$

\section{Simulation and Experimental Results}

The experimental setup for this study is shown in Figure 7 which consists of one-degree-of-freedom EHA system with an electronically controlled servo valve. Tracking performance of the EHA system with an inertial load of $15 \mathrm{~kg}$ is implemented with the developed controller. Simulation studies are conducted first based on the identified nonminimum phase discrete-time model which is purposely to ensure that the proposed controller is properly designed.

For the overall controller design as shown in Figure 8, the feedforward is represented by ZPETC where its function is approximation of the inverse closed-loop system with LQR controller. Output from the nonminimum phase of EHA

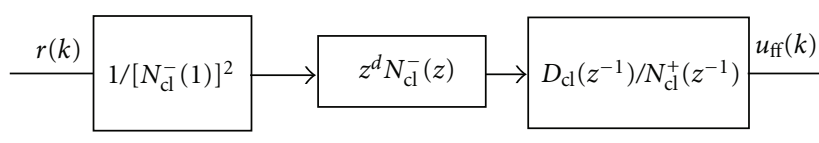

Figure 6: ZPETC block diagram.

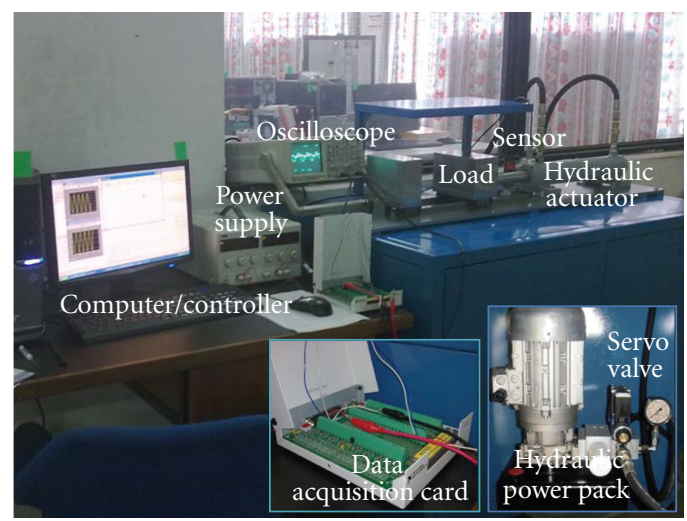

FIGURE 7: Experimental workbench.

system is fed into the LQR controller. The LQR controller operated as a feedback control which permits the system's output to follow the desired trajectory. The proposed ZPETC based on inverse closed-loop system is introduced to reduce the phase lag due to feedback control problem. Figure 9 shows the output of tracking controller from simulation and experimental study, while its control signals are represented in Figure 10. It clearly shows that the linear model is capable to represent the EHA system, and the proposed controller achieved good accuracy in $30 \mathrm{~mm}$ reference trajectory. The experimental results also show that the tracking output and its control signal are following slightly the performance of the simulation results. The proposed controller has been finetuned in this simulation study before the implementation with real systems.

In the experimental study, proportional-integral-derivative (PID) controller is used as a comparison to the proposed LQR with ZPETC controller. The PID controller without a feedforward controller is designed using ZieglerNichols technique. A random of sinusoidal signal is used as a reference trajectory to show the capability in reducing phase error using LQR with ZPETC controller. Figure 11 shows the performance of tracking control using LQR with ZPETC controller as compared with conventional PID controller. It shows that the phase lag problem occurs during the tracking control can be reduced by the proposed controller. The tracking error shows significant difference between the desired trajectory and the actual output in Figure 12 by introducing the ZPETC controller. The phase lag is significantly reduced in the tracking control process. However, the optimal control using LQR still needs an improvement where the parameter is needed to be tuned on line due to the nonlinearities and disturbances exist in the EHA system. Therefore, a robust control is needed to encounter these difficulties. 


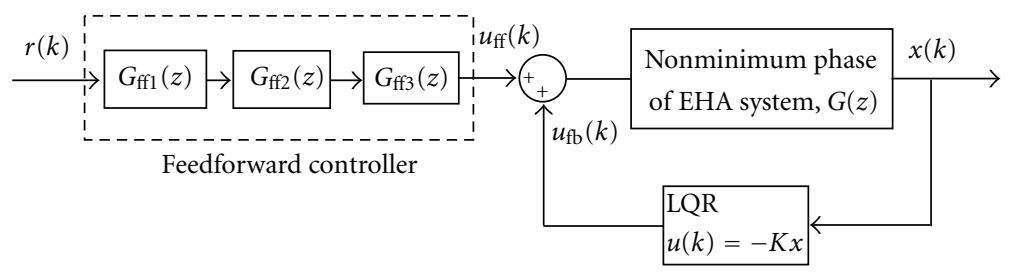

Feedback controller

Figure 8: Proposed controller design.

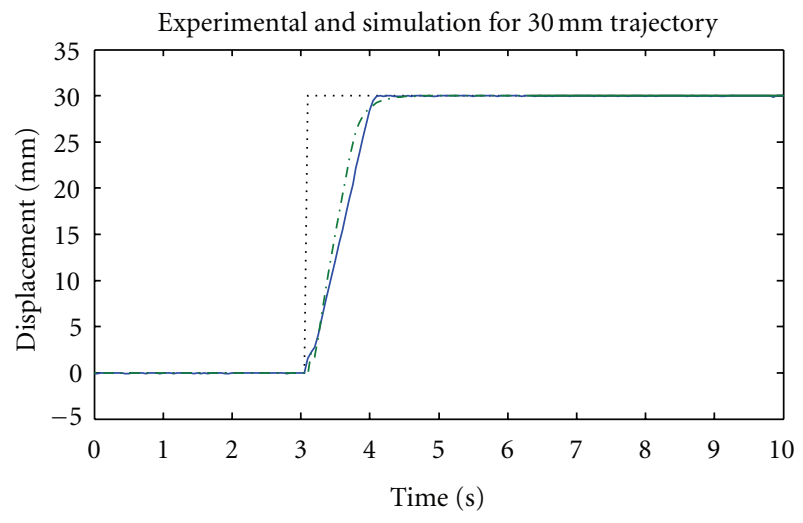

.... Reference

- Experimental

-..- Simulation

FIGURE 9: Simulation and experimental results of $30 \mathrm{~mm}$ reference trajectory.

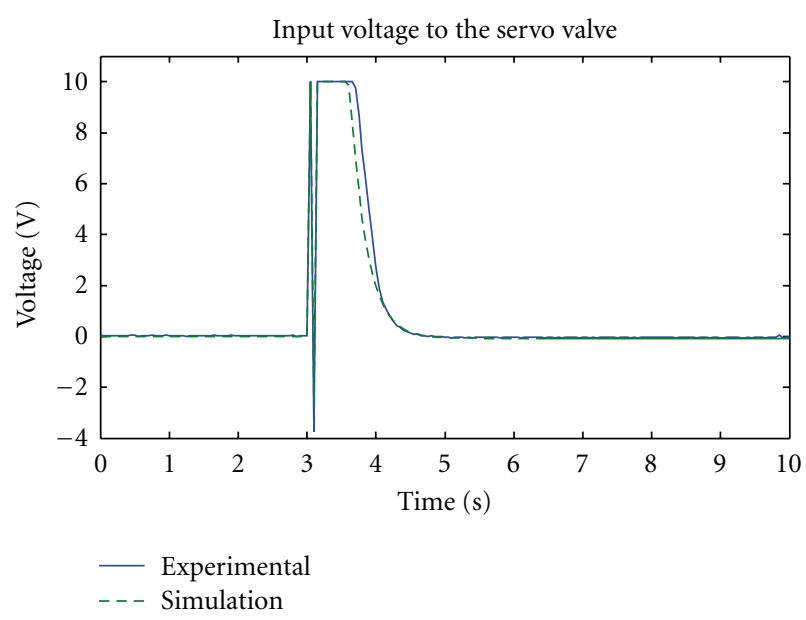

FIGURE 10: Input signal from the controller in simulation and experimental.

\section{Conclusion}

In this study, the feedforward controller is developed based on ZPETC technique to reduce the phase lag emerged by
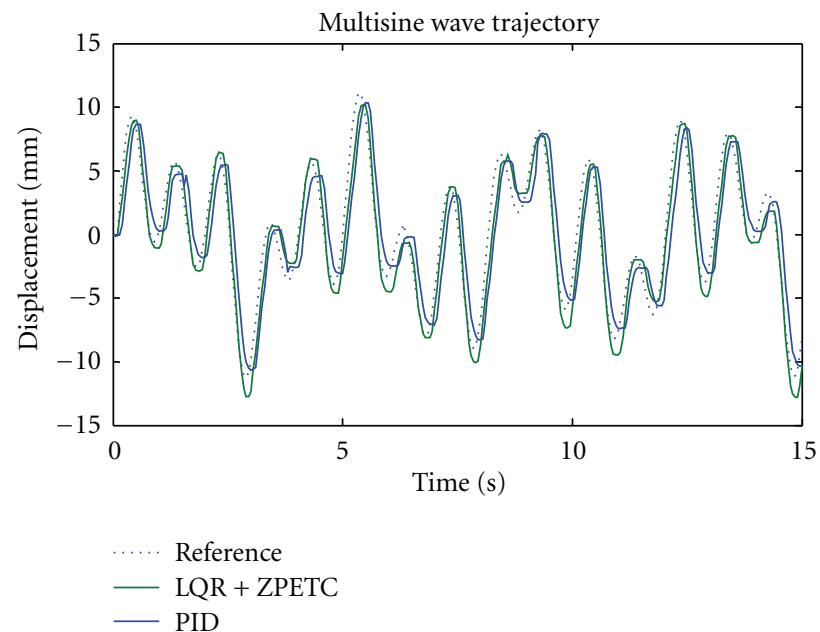

Figure 11: Position tracking results (experimental).

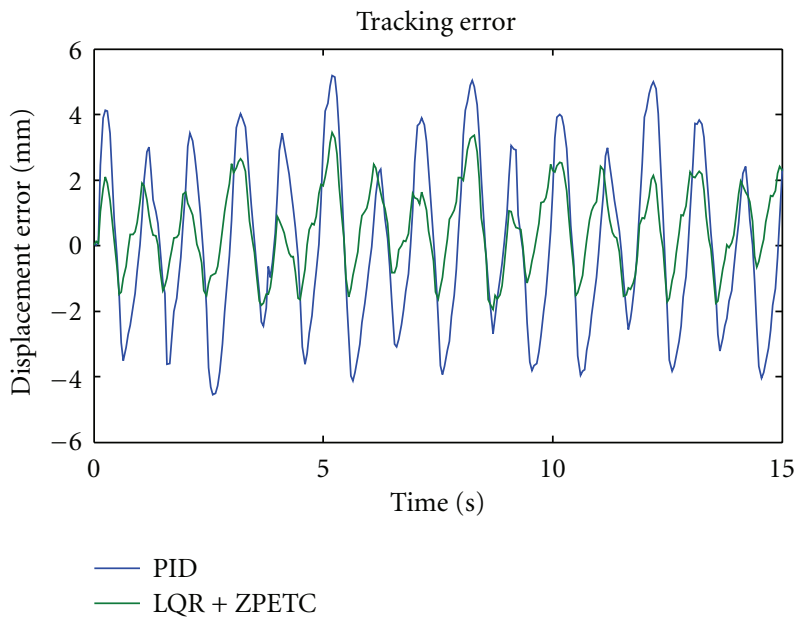

Figure 12: Position tracking error (experimental).

feedback controller during the tracking control. An approximation of the inverse closed-loop system in the feedforward controller design is adopted due to nonminimum phase problem in the EHA system model. It is shown that the controller offers good performance in reducing phase and gain error that usually occur in positioning or tracking systems. 
As expected and verified by experiment, better performance is achieved for the proposed controller compared with PID controller, thus signifying its utility for high-performance control design. As conclusion, the proposed controller design using ZPETC is particularly suited to the various positioning control applications that encounter nonminimum phase problem to achieve perfect tracking control. The realization of a state feedback controller can be made for a single-output feedback control problem. As future work, a robust control is needed to replace the optimal control to encounter the nonlinearities and uncertainties exist during the tracking control.

\section{Acknowledgments}

The authors would like to thank Universiti Teknologi Malaysia (UTM), Universiti Tun Hussein Onn Malaysia (UTHM), and Ministry of Science, Technology and Innovation (MOSTI), Malaysia for their support.

\section{References}

[1] H. E. Merrit, Hydraulic Control Systems, John Wiley \& Sons, New York, NY, USA, 1967.

[2] M. Karpenko and N. Sepehri, "Hardware-in-the-loop simulator for research on fault tolerant control of electrohydraulic actuators in a flight control application," Mechatronics, vol. 19, no. 7, pp. 1067-1077, 2009.

[3] J. C. Renn and C. Tsai, "Development of an unconventional electro-hydraulic proportional valve with fuzzy-logic controller for hydraulic presses," International Journal of Advanced Manufacturing Technology, vol. 26, no. 1-2, pp. 10-16, 2005.

[4] M. H. Chiang, Y. P. Yeh, F. L. Yang, and Y. N. Chen, "Integrated control of clamping force and energy-saving in hydraulic injection moulding machines using decoupling fuzzy slidingmode control," International Journal of Advanced Manufacturing Technology, vol. 27, no. 1-2, pp. 53-62, 2005.

[5] S. Jha and V. K. Jain, "Design and development of the magnetorheological abrasive flow finishing (MRAFF) process," International Journal of Machine Tools and Manufacture, vol. 44, no. 10, pp. 1019-1029, 2004.

[6] J. Ruan, X. Pei, and M. Zhu, "Identification and modeling of electrohydraulic force control of the material test system (MTS)," Journal of Physics: Conference Series, vol. 48, no. 1, pp. 1322-1326, 2006.

[7] M. H. Chiang and C. C. Huang, "Experimental implementation of complex path tracking control for large robotic hydraulic excavators," International Journal of Advanced Manufacturing Technology, vol. 23, no. 1-2, pp. 126-132, 2004.

[8] S. W. Lo and T. C. Yang, "Closed-loop control of the blank holding force in sheet metal forming with a new embeddedtype displacement sensor," International Journal of Advanced Manufacturing Technology, vol. 24, pp. 553-559, 2004.

[9] M. C. Wu and M. C. Shih, "Simulated and experimental study of hydraulic anti-lock braking system using sliding-mode PWM control," Mechatronics, vol. 13, no. 4, pp. 331-351, 2003.

[10] Y. M. Sam, J. H. S. Osman, and M. R. A. Ghani, "A class of proportional-integral sliding mode control with application to active suspension system," Systems and Control Letters, vol. 51, no. 3-4, pp. 217-223, 2004.
[11] A. R. Plummer, "Model-in-the-loop testing," Journal of Systems and Control Engineering, vol. 220, no. 3, pp. 183-199, 2006.

[12] T. Knohl and H. Unbehauen, "Adaptive position control of electrohydraulic servo systems using ANN," Mechatronics, vol. 10, no. 1-2, pp. 127-143, 2000.

[13] Zulfatman and M. F. Rahmat, "Application of self-tuning Fuzzy PID controller on industrial hydraulic actuator using system identification approach," International Journal on Smart Sensing and Intelligent Systems, vol. 2, no. 2, pp. 246261, 2009.

[14] Ş. Çetin and A. V. Akkaya, "Simulation and Hybrid FuzzyPID control for positioning of a hydraulic system," Nonlinear Dynamics, vol. 61, no. 3, pp. 465-476, 2010.

[15] K. Ziaei and N. Sepehri, "Design of a nonlinear adaptive controller for an electrohydraulic actuator," Journal of Dynamic Systems, Measurement and Control, vol. 123, no. 3, pp. 449456, 2001.

[16] A. Kireçci, M. Topalbekiroglu, and I. Eker, "Experimental evaluation of a model reference adaptive control for a hydraulic robot: a case study," Robotica, vol. 21, no. 1, pp. 71-78, 2003.

[17] N. Sepehri and G. Wu, "Experimental evaluation of generalized predictive control applied to a hydraulic actuator," Robotica, vol. 16, no. 4, pp. 463-474, 1998.

[18] R. Ghazali, Y. M. Sam, M. F. Rahmat, A. W. I. M. Hashim, and Zulfatman, "Sliding mode control with PID sliding surface of an electro-hydraulic servo system for position tracking control," Australian Journal of Basic and Applied Sciences, vol. 4, no. 10, pp. 4749-4759, 2010.

[19] J. Wang, H. Van Brussel, and J. Swevers, "Robust perfect tracking control with discrete sliding mode controller," Journal of Dynamic Systems, Measurement and Control, vol. 125, no. 1, pp. 27-32, 2003.

[20] K. J. Astrom and B. Wittenmark, Computer-Controlled System: Theory and Design, Prentice Hall, Englewood Cliffs, NJ, USA, 1997.

[21] M. Tomizuka, "Zero phase error tracking algorithm for digital control," Journal of Dynamic Systems, Measurement and Control, vol. 190, no. 1, pp. 65-68, 1987.

[22] M. M. Mustafa, "Trajectory-adaptive digital tracking controllers for non-minimum phase systems without factorisation of zeros," IEE Proceedings: Control Theory and Applications, vol. 149, no. 2, pp. 157-162, 2002.

[23] M. Kalyoncu and M. Haydim, "Mathematical modelling and fuzzy logic based position control of an electrohydraulic servosystem with internal leakage," Mechatronics, vol. 19, no. 6, pp. 847-858, 2009.

[24] I. Eker, "Experimental on-line identification of an electromechanical system," ISA Transactions, vol. 43, no. 1, pp. 13-22, 2004.

[25] A. R. Plummer and N. D. Vaughan, "Robust adaptive control for hydraulic servosystems," Journal of Dynamic Systems, Measurement and Control, vol. 118, no. 2, pp. 237-244, 1996.

[26] I. Eker, "Open-loop and closed-loop experimental on-line identification of a three-mass electromechanical system," Mechatronics, vol. 14, no. 5, pp. 549-565, 2004.

[27] A. Saleem, S. Abdrabbo, and T. Tutunji, "On-line identification and control of pneumatic servo drives via a mixed-reality environment," International Journal of Advanced Manufacturing Technology, vol. 40, no. 5-6, pp. 518-530, 2009. 

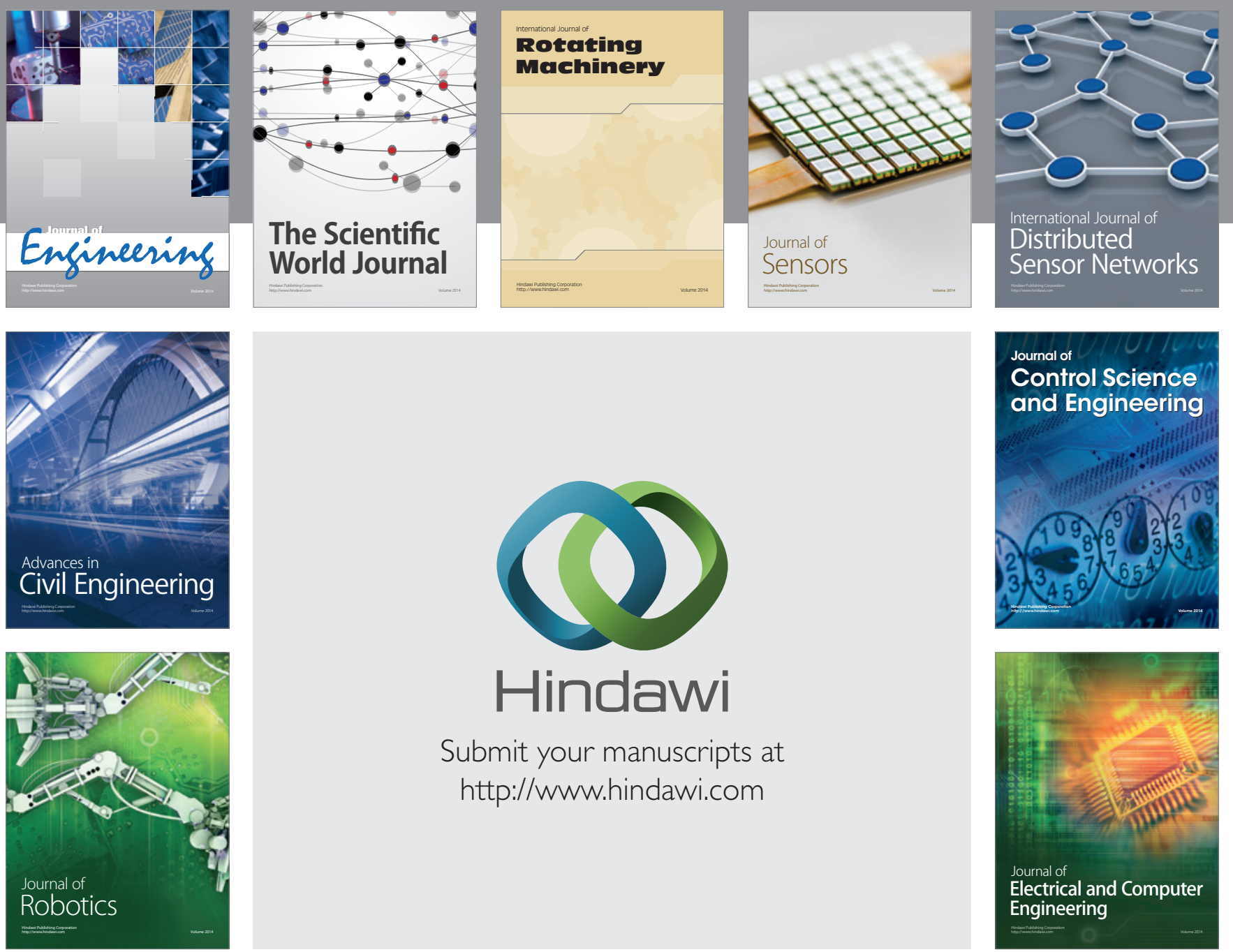

Submit your manuscripts at

http://www.hindawi.com
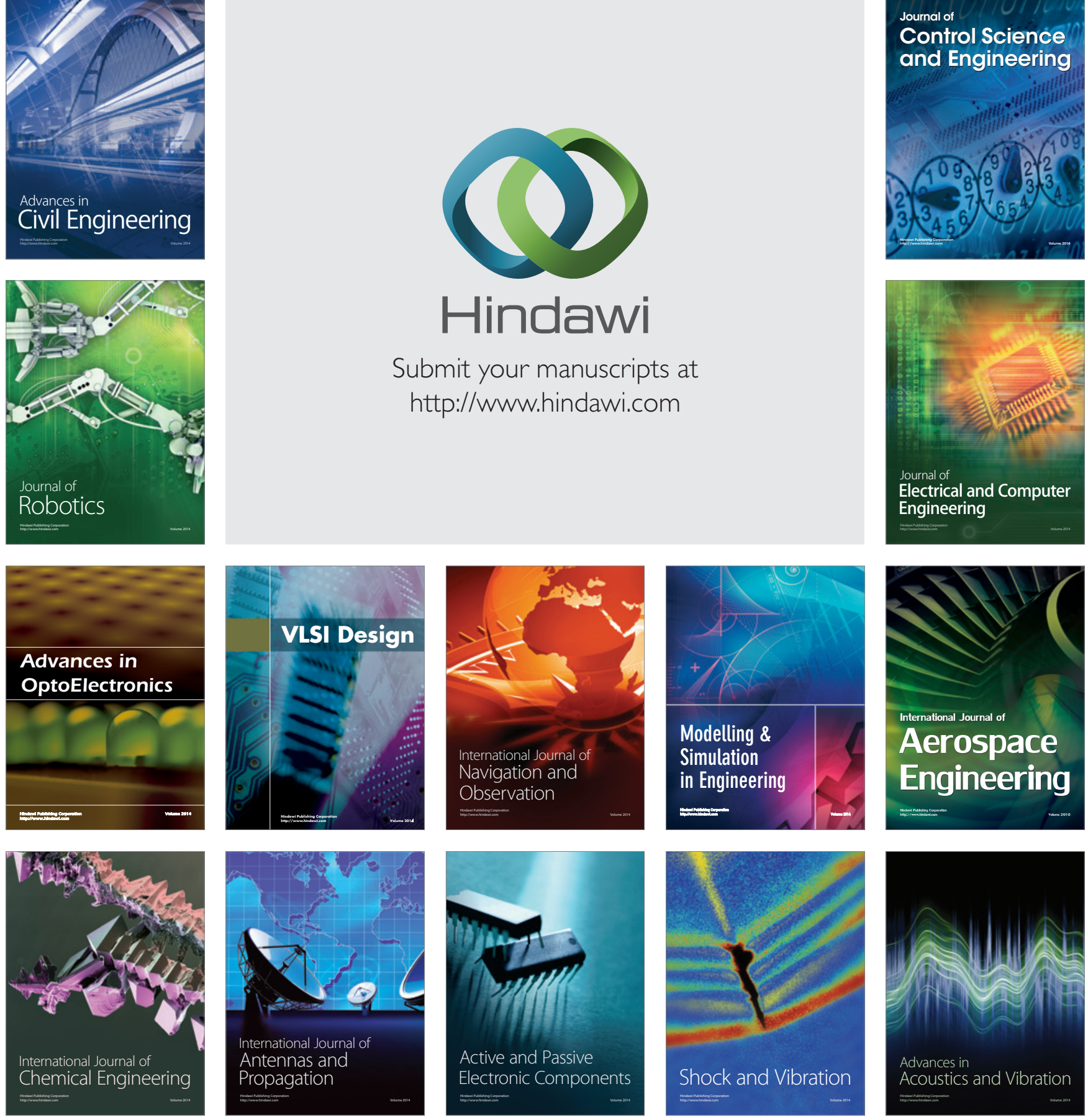